
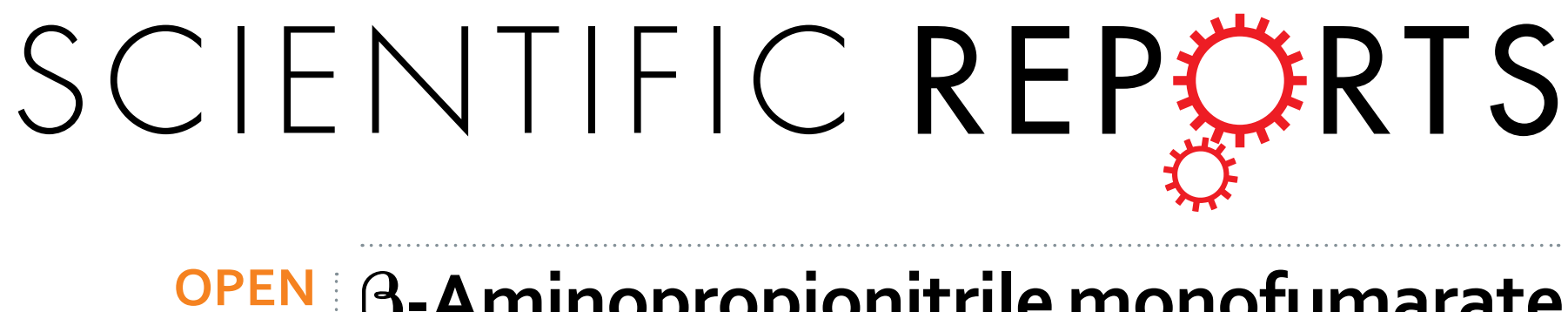

\title{
$\beta$-Aminopropionitrile monofumarate induces thoracic aortic dissection in C57BL/6 mice
}

Received: 16 January 2016

Accepted: 01 June 2016

Published: 22 June 2016
Weihong Ren", Yan Liu*, Xuerui Wang, Lixin Jia, Chunmei Piao, Feng Lan \& Jie Du,

Thoracic aortic dissection (TAD) is a catastrophic disease with high mortality and morbidity, characterized by fragmentation of elastin and loss of smooth muscle cells. However, the underlying pathological mechanisms of this disease remain elusive because there are no appropriate animal models, limiting discovery of effective therapeutic strategies. We treated mice on C57BL/6 and FVB genetic backgrounds with $\beta$-aminopropionitrile monofumarate (BAPN), an irreversible inhibitor of lysyl oxidase, for $4 \mathrm{wk}$, followed by angiotensin II (Ang II) infusion for $24 \mathrm{~h}$. We found that the BAPN plus Ang II treatment induced formation of aortic dissections in $100 \%$ of mice on both genetic backgrounds. BAPN without Ang II caused dissections in few FVB mice, but caused $87 \%$ of $\mathrm{C} 57 \mathrm{BL} / 6$ mice to develop TAD, with $37 \%$ dying from rupture of the aortic dissection. Moreover, a lower dose of BAPN induced TAD formation and rupture earlier with fewer effects on body weight. Therefore, we have generated a reliable and convenient TAD model in C57BL/6 mice for studying the pathological process and exploring therapeutic targets of TAD.

Thoracic aortic dissection (TAD) ranks among the most lethal vascular diseases, occurring at a rate of 3 cases per 100,000 individuals per year. Blood enters into the media space of thoracic aorta, leading to separation of the layers within the aortic wall, which characterizes TAD. Its key pathologic feature is medial degeneration, a process characterized by smooth muscle cell depletion and extracellular matrix degradation ${ }^{1}$. However, the molecular mechanisms of medial degeneration remain elusive because there is no stable, convenient model of TAD 2 .

Most of the existing mouse models reported for TAD were caused by genetic mutation, such as in fibrillin-1, TGBBR, SMAD3 or ACTA2 $2^{3,4}$. Besides these genetic models, surgery and drug treatment have also been used to induce TAD. For example, either long-term angiotensin II (Ang II) infusion using apoE-/- mice or continuous perfusion of elastase, resulted in abdominal aortic aneurysm (AAA) or abdominal aortic dissection (AAD) ${ }^{5-7}$. However, the rate of TAD induction is very low in these models. Thus, there is no appropriate TAD mouse model for studying the mechanism of TAD.

Recently, it was reported that a 4 -wk administration of $\beta$-aminopropionitrile monofumarate (BAPN), an inhibitor of lysyl oxidase (LOX), combined with a 24-h infusion of Ang II, induced aortic dissection (AD) rupture in wildtype FVB mice, while BAPN administration alone caused dissection in about $10 \%$ of these mice . $^{8}$. In another study, LOX-null C57BL/6 mice developed aortic rupture spontaneously ${ }^{9,10}$ and in our recent report, BAPN administration alone induced TAD in wildtype C57BL/6 mice 4 . In this study, we systemically evaluated how BAPN can induce TAD in mice and examined its pathologic characteristics.

Results

Basal characterization of mice with BAPN treatment, with or without Ang II. It was reported that treatment with the LOX inhibitor BAPN plus Ang II induced TAD in FVB mice ${ }^{8}$. We recently found that, on a C57BL/6 background, the same dose of BAPN caused sudden death in approximately $56 \%$ of mice, prior to Ang II administration, and that this was caused by thoracic aortic ruptures ${ }^{4}$. We thus compared the effects of BAPN in mice with the two genetic backgrounds. Male mice ( 3 wk old) on FVB or C57BL/6 backgrounds were administered BAPN in drinking water at a dose of $1 \mathrm{~g}$ per kg body weight for $4 \mathrm{wk}$. BAPN treatment reduced diastolic (Fig. 1a) with no effect on systolic (Fig. 1b) blood pressure, indicating increased aortic stiffness. BAPN treatment

Beijing Anzhen Hospital, Affiliated to Capital Medical University; Collaborative Innovation Center for Cardiovascular Disorders; The Key Laboratory of Remodeling-Related Cardiovascular Diseases, Ministry of Education; Beijing Institute of Heart Lung and Blood Vessel Diseases, Beijing 100029, China. *These authors contributed equally to this work. Correspondence and requests for materials should be addressed to J.D. (email: jdu@bcm.edu) 
a

b
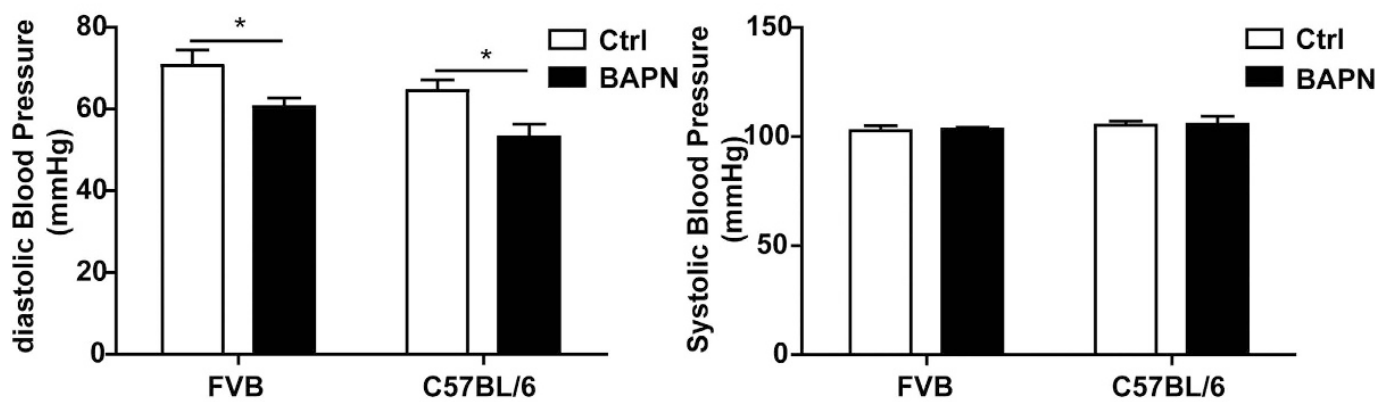

C

\section{d}

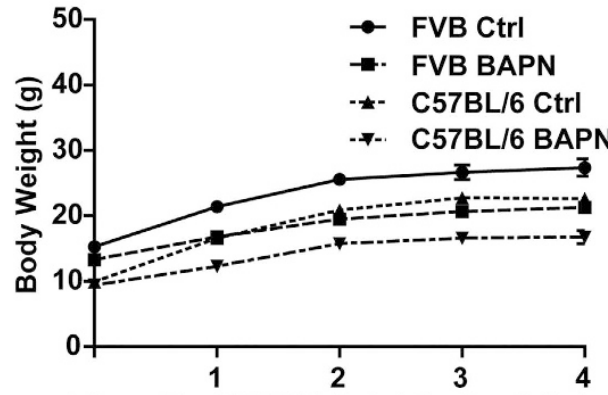

Time after BAPN Administration (wks)

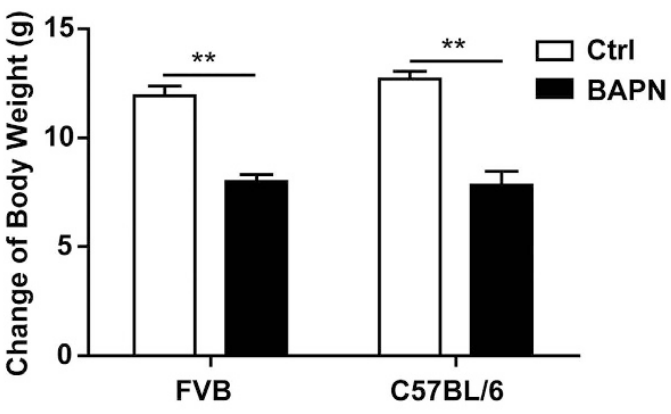

e

f
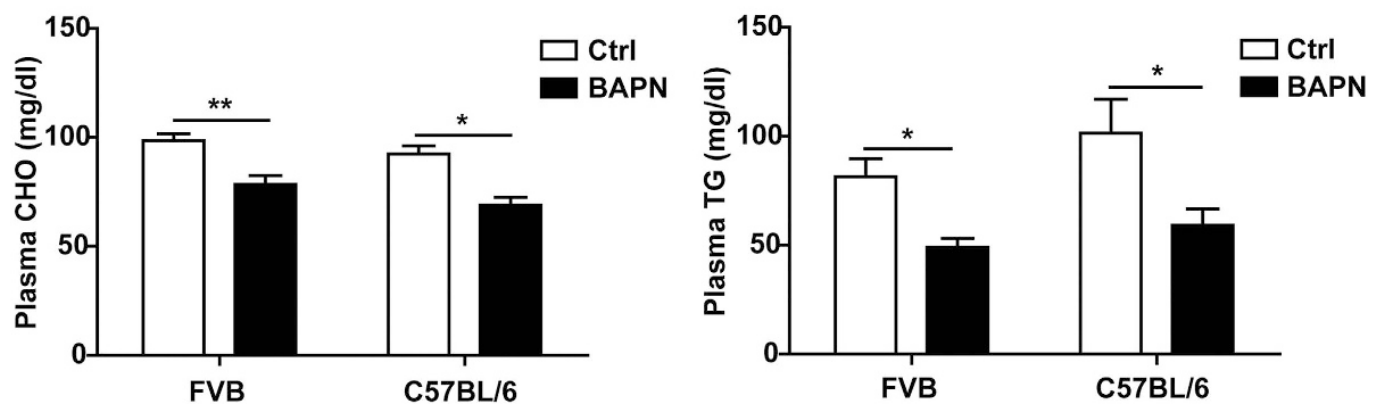

Figure 1. Basal parameters of BAPN treatment. C57BL/6 and FVB mice were administered vehicle or BAPN for 4 wk. (a,b) Diastolic and systolic blood pressures ( $\mathrm{n}=6$ per group); $* P<0.05$. (c,d) Body weights with bar graph showing changes during the study $(\mathrm{n}=6$ per group); $* * P<0.01$. (e,f) Levels of plasma cholesterol $(\mathrm{CHO})$ $(\mathrm{n}=11$ for FVB Ctrl, $\mathrm{n}=16$ for FVB BAPN, $\mathrm{n}=6$ for C57BL/ 6 Ctrl, $\mathrm{n}=15$ for C57BL/6 BAPN) and triglyceride (TG) $(\mathrm{n}=12$ for FVB Ctrl, $\mathrm{n}=6$ for FVB BAPN, $\mathrm{n}=7$ for C57BL/ 6 Ctrl, $\mathrm{n}=15$ for C57BL/6 BAPN); $* P<0.05$, $* * P<0.01$.

also attenuated body weight gains (Fig. 1c,d) and significantly decreased plasma triglyceride and cholesterol levels (Fig. 1e,f) in both FVB and C57BL/6 mice.

Induction of TAD with BAPN treatment. We next examined the effects of BAPN treatment on the incidence of TAD. To determine whether Ang II was also required for TAD development, the mice were sacrificed for autopsy after $4 \mathrm{wk}$ of BAPN treatment, with or without $24 \mathrm{~h}$ of Ang II infusion. Consistent with the previous report $^{8}$, administration of BAPN plus Ang II induced TAD in all mice, while approximately 75\% of FVB mice treated with BAPN alone did not develop TAD (Fig. 2a and Table 1). However, the incidence of TAD in C57BL/6 mice treated with only BAPN reached $87 \%$ (Fig. 2 a and Table 1 ) and $45 \%$ of mice in this group died of aortic rupture. TAD was also observed in all C57BL/6 mice treated with BAPN plus Ang II, with 50\% of these having aortic rupture within $24 \mathrm{~h}$ of Ang II infusion. Aortas of C57BL/6 mice given BAPN, with or without Ang II infusion, were enlarged from the root to the thoracic segment and, in some cases, the abdominal segment was also involved. Hematomas were observed in the lesions, indicating thrombosis (Fig. 2a). Haematoxylin and eosin staining showed tearing of the aortic wall and thrombi in the false lumens (Fig. 2b). Massive fragmentation and depletion of elastic fibres and smooth muscle cell loss were confirmed by Elastica van Gieson (EVG) staining (Fig. 2c). All these pathological changes resembled those observed histopathologically in humans (Fig. 2d). 
a

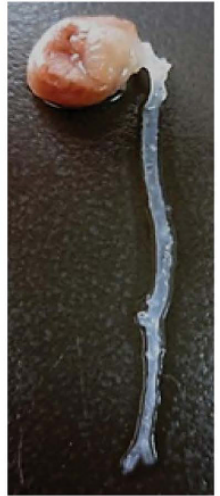

Ctrl

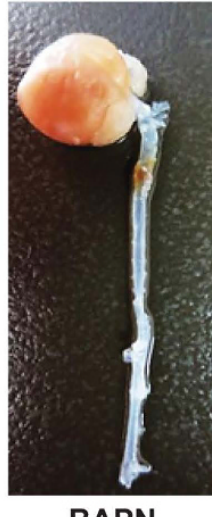

BAPN

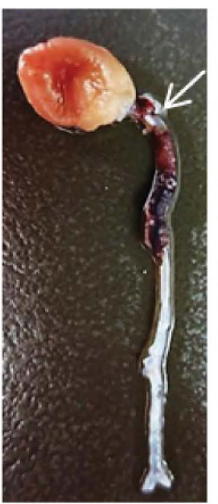

BAPN+Angll

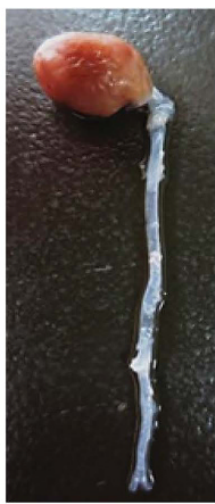

Ctrl

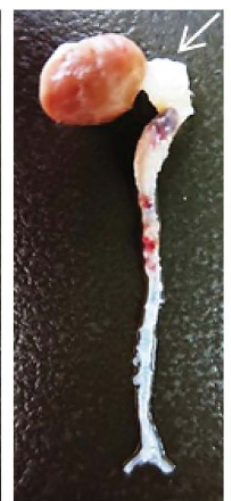

BAPN

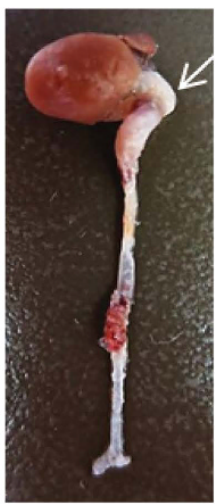

BAPN+Angll

b FVB

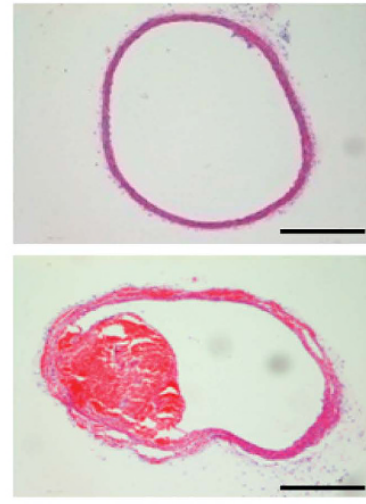

BAPN

\section{C57BL/6}
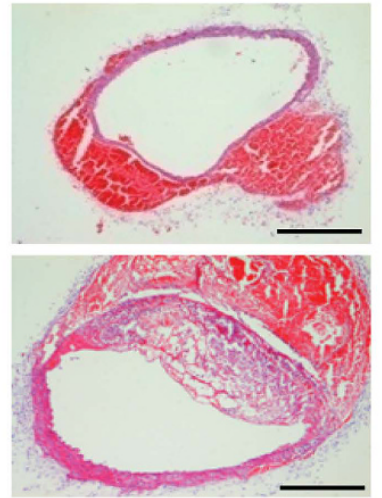

BAPN+Angll

C

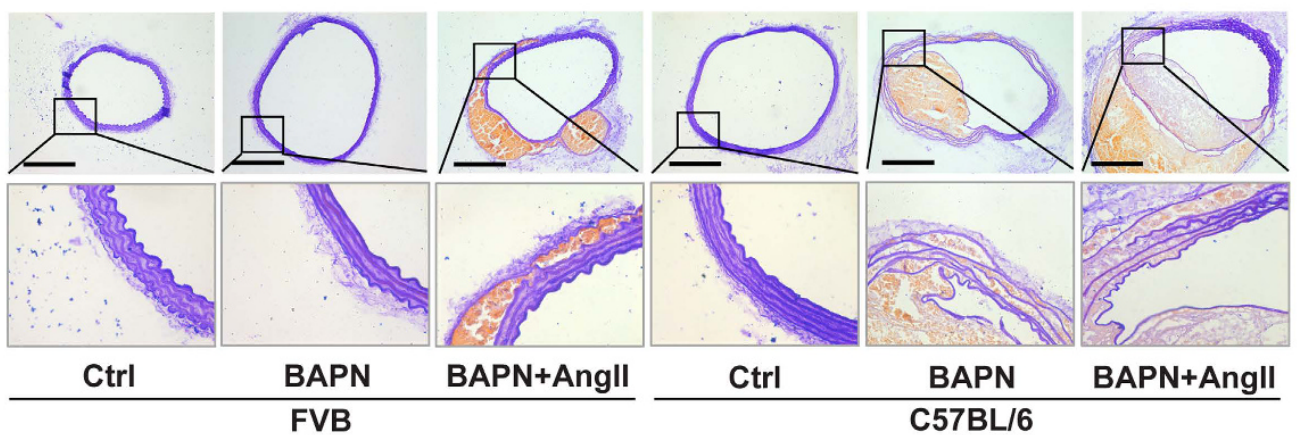

d

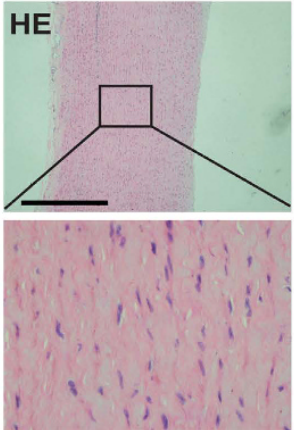

Ctrl
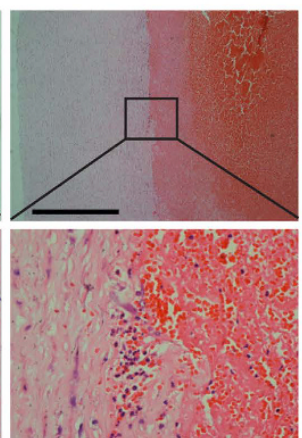

TAD

e

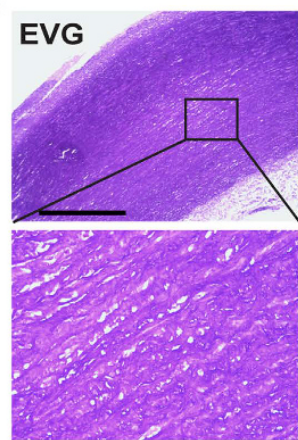

Ctrl

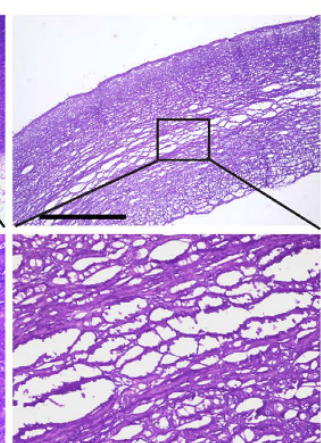

TAD

Figure 2. BAPN treatment, without Ang II infusion, induced TAD in C57BL/6 mice, reproducing major features of human TAD. (a) Representative images showing macroscopic features of isolated mouse aorta after vehicle or BAPN treatment for 4 wk; white arrow indicates location of TAD. (b) Representative haematoxylin and eosin (HE) staining showing blood clot inside torn aortic wall after BAPN administration. (c) Representative Elastica Van Gieson (EVG) staining presenting elastic fragmentation and loss of smooth muscle cells after BAPN administration. Representative HE (d) and EVG (e) staining in human samples. Scale bar: $50 \mu \mathrm{m}$. 


\begin{tabular}{|l|c|c|c|c|}
\hline \multirow{2}{*}{} & \multicolumn{2}{|c|}{ BAPN } & \multicolumn{2}{c|}{ BAPN + AngII } \\
\cline { 2 - 5 } & FVB & C57BL/6 & FVB & C57BL6 \\
\hline AD & $25 \%$ & $87 \%$ & $100 \%$ & $100 \%$ \\
\hline TAD & $25 \%$ & $87 \%$ & $100 \%$ & $75 \%$ \\
\hline AAD & $25 \%$ & $13 \%$ & $20 \%$ & $75 \%$ \\
\hline AD-rupture & $0 \%$ & $37 \%$ & $20 \%$ & $50 \%$ \\
\hline
\end{tabular}

Table 1. Incidence of TAD in mice treated with BAPN, with or without Ang II. AD, aortic dissection; TAD, thoracic aortic dissection; AAD, abdominal aortic dissection.

a

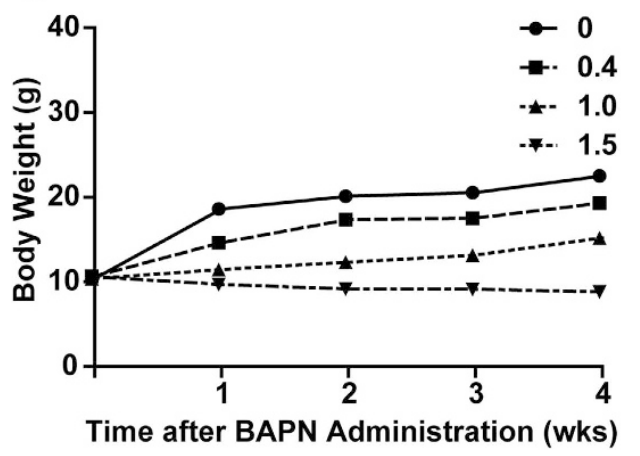

b

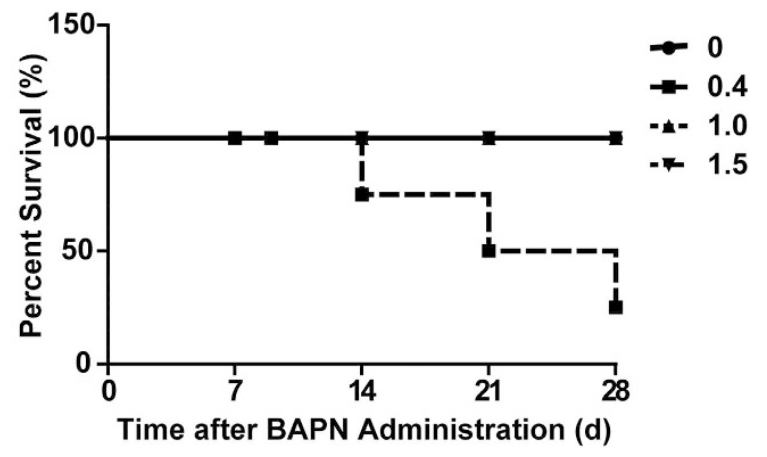

Figure 3. Dose-dependence of BAPN-induced TAD in C57BL/6 mice. C57BL/6 mice were equally segregated into four weight-matched groups and administered diets containing $0,0.4,1.0$ or $1.5 \mathrm{~g}$ BAPN per $100 \mathrm{~g}$ diet, respectively, for $4 \mathrm{wk}$ ( $\mathrm{n}=6$ per group). (a) Body weights measured weekly. (b) Kaplan-Meier survival curves.

BAPN dose optimization for TAD induction. To further investigate the causal effects of medial degeneration on TAD formation, we applied different doses of BAPN by feeding 3-wk-old C57BL/6 male mice with diets containing $0,0.4,1.0$ or $1.5 \mathrm{~g}$ BAPN per $100 \mathrm{~g}$ mouse chow for $4 \mathrm{wk}$. Body weights were lower with increased BAPN doses (Fig. 3a). All six mice fed with the $0.4 \mathrm{~g}$ BAPN per $100 \mathrm{~g}$ diet developed TAD and five died of dissection ruptures by 2 to $4 \mathrm{wk}$ after BAPN administration. Of six mice fed with the $1.0 \mathrm{~g}$ BAPN diet, two had TAD at the end of the treatment, but no ruptures occurred. Most surprisingly, no TAD formation was observed in mice fed with the $1.5 \mathrm{~g}$ BAPN diet (Fig. 3b).

Molecular phenotypic features of BAPN-induced TAD. Because BAPN-induced TAD exhibited typical histological features of the human disease, we next examined whether expression of TAD-related genes were also changed in the media of aortas. A panel of genes known to be dysregulated during medial degradation in TAD formation were selected for analysis. These were matrix metalloproteinases (MMPs, MMP2/3/9) ${ }^{5,8,11,12}$ and cathepsins (cathepsin $\mathrm{S} / \mathrm{K} / \mathrm{L})^{13}$ (that degrade extracellular matrix), collagen I $\alpha 1$ (COL1 $\left.\alpha 1\right)$ and connective tissue growth factor (CTGF) (target genes indicating activation of the TGF- $\beta$ signalling pathway in LDS ${ }^{14}$, $\alpha$-smooth muscle actin ( $\alpha$-SMA) and $\beta$-myosin heavy chain ( $\beta$-MHC) (associated with familial thoracic aortic aneurysm and dissection syndrome $)^{15}$. Expression of these genes was compared in control and BAPN-treated C57BL/6 mice. In the BAPN-treated group, compared with the control, MMP2 was significantly upregulated (Fig. 4a), while MMP3 and MMP9 were downregulated (Fig. 4b,c). Cathepsin S and cathepsin K levels were no different in the two groups (Fig. 4d,e), while cathepsin L was significantly decreased in the BAPN group (Fig. 4f). Both COL1 $\alpha 1$ and $\alpha$-SMA expression were dramatically decreased with BAPN treatment (Fig. 4g,h), while CTGF and $\beta$-MHC levels were not changed (Fig. 4i,j). These results revealed that BAPN-induced TAD was associated with typical ECM degradation, possibly via MMP2, and loss of SMC leading to decreased $\alpha$-SMA, effects consistent with previous observations in humans and mouse models.

\section{Discussion}

Thoracic aortic dissection, with or without rupture, represents a structural and functional failure of the aortic wall and can occur when integrity of the thoracic aortic wall is impaired ${ }^{15}$. In our study, we found that at the proper dose $(0.4 \mathrm{~g}$ BAPN per $100 \mathrm{~g}$ diet), BAPN disrupted the aortic medial layer without substantially affecting metabolism and body growth, effectively inducing TAD in C57BL/6 mice, a widely used strain. We showed that TAD in C57BL/6 mice resembled clinically observed TAD, with a high incidence of rupture and mortality.

Clinically, TAD, without exception, exhibits medial degeneration, which results from aortic wall stressors such as hypertension, genetics and inflammatory conditions ${ }^{15}$. Medial degeneration is, therefore, essential for rupture of the intima or haemorrhage within the media. Thus, acute blood pressure elevation, such as hypertension induced by Ang II perfusion, is likely to trigger, but not serve as a pathological mediator of, TAD and rupture. Moreover, in a previous report, norepinephrine infusion in BAPN-treated mice failed to induce TAD, 
a

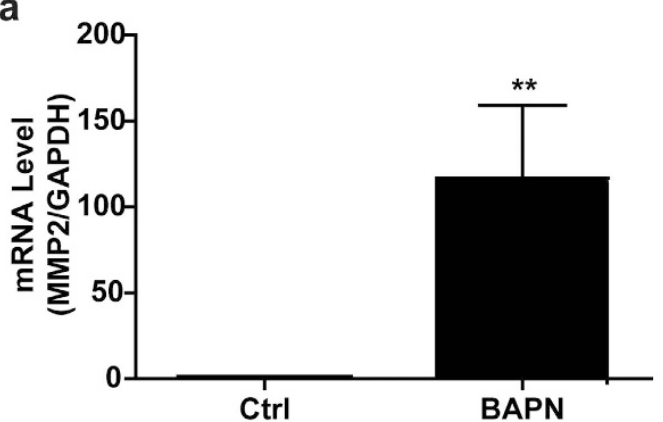

C
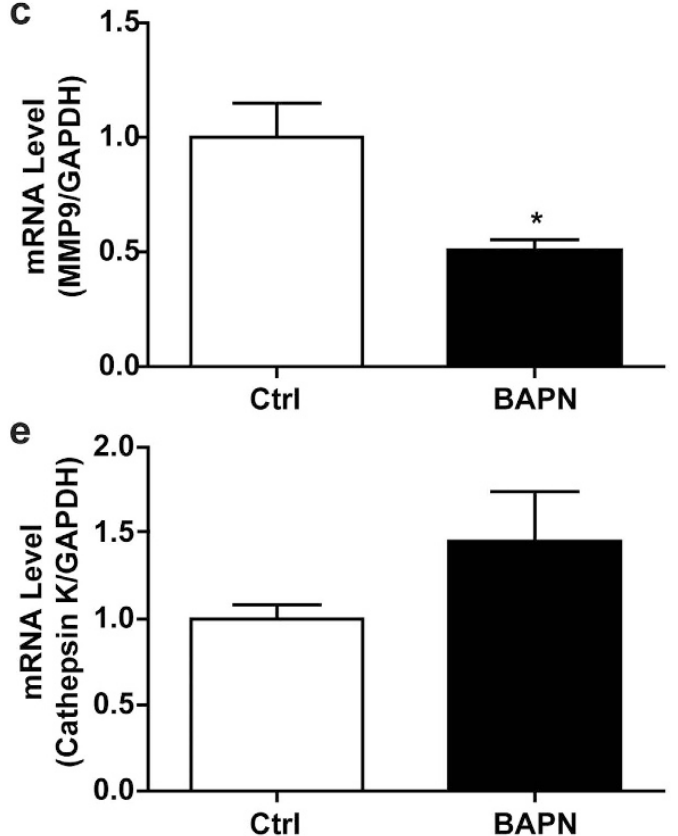

g
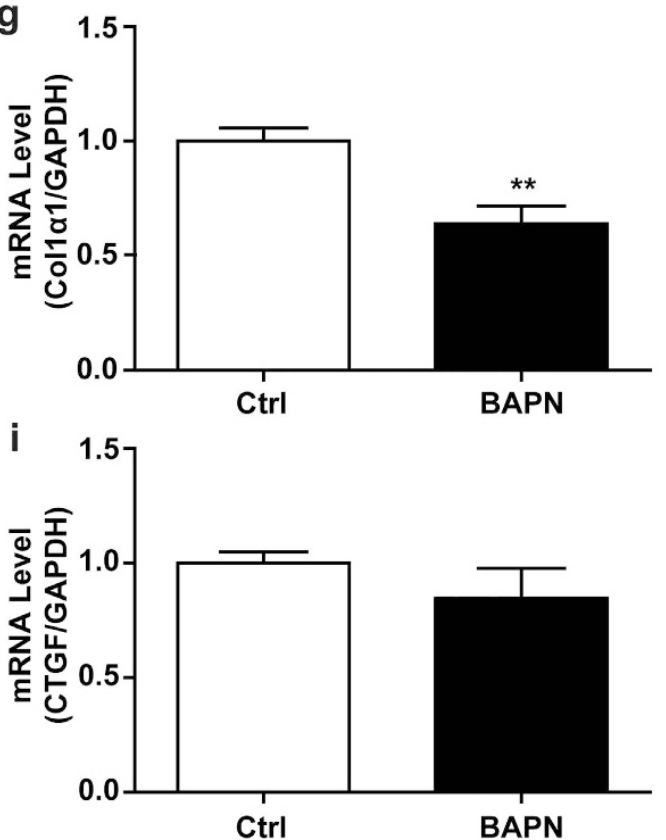

b
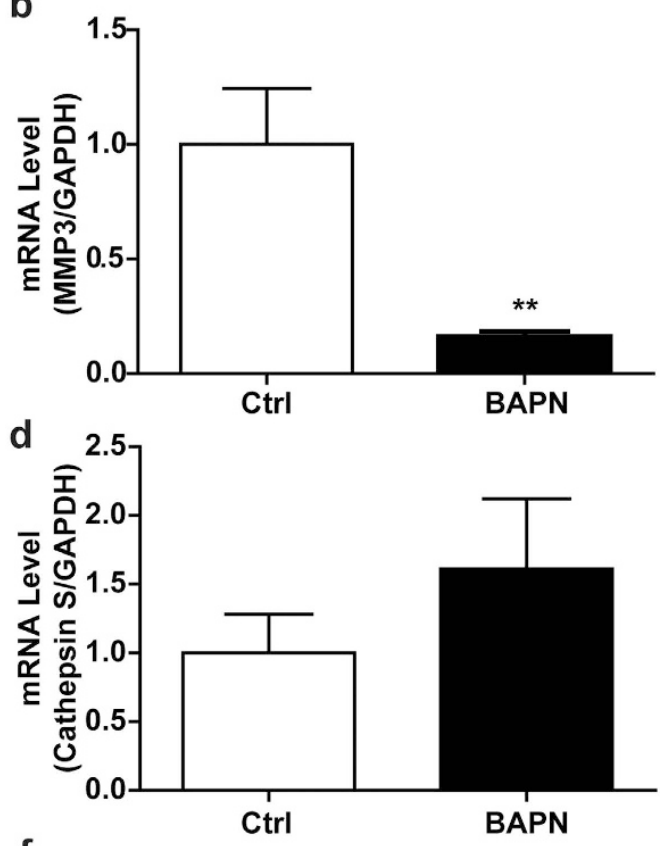

f

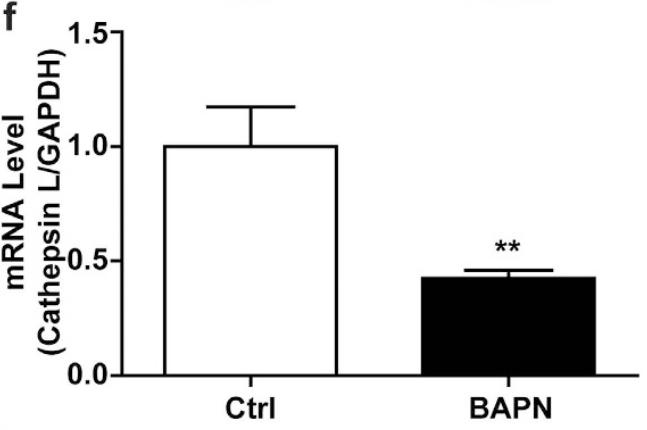

h
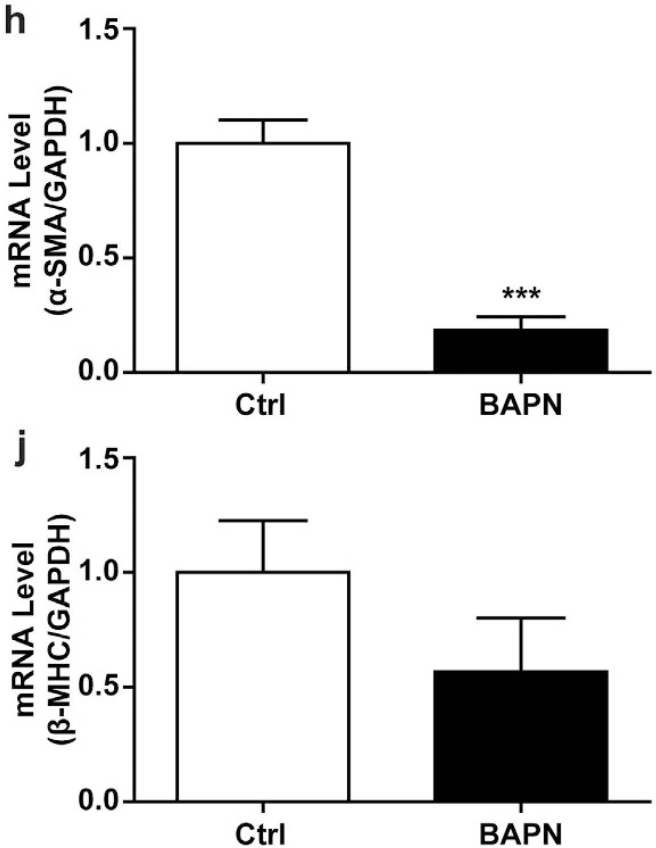

Figure 4. Phenotype-related gene expression in aortic media of BAPN-treated mice. C57BL/ 6 mice were administered vehicle or BAPN for $4 \mathrm{wk}$. (a-c) qPCR analysis of MMP2/3/9 mRNA levels; $\mathrm{n}=8, * P<0.05, * * P<0.01$. (d-f) qPCR analysis of Cathepsin S/K/L mRNA levels ( $\mathrm{n}=8$ per group); $* P<0.05$, $* * P<0.01$. (g-j) qPCR analysis of COL1 $11, \alpha$-SMA, CTGF and $\beta$-MHC mRNA levels ( $\mathrm{n}=8$ per group); $* P<0.05, * * P<0.01, * * * P<0.0001$. 


\begin{tabular}{|c|c|}
\hline Gene name & Oligoes for primer (forward/reverse) \\
\hline mouse MMP2 & 5'-CGATGTCGCCCCTAAAACAG-3' 5'-GCATGGTCTCGATGGTGTTC-3' \\
\hline mouse MMP3 & 5'-GTTCTGGGCTATACGAGGGC-3' 5'-TTCTTCACGGTTGCAGGGAG-3' \\
\hline mouse MMP9 & 5'-TGGGCGTTAGGGACAGAAAT-3' 5'-GAACCATAACGCACAGACCC-3' \\
\hline mouse Cathepsin S & 5'-CATCTTTGGAGTGAGCACCA-3' 5' -GCATCCAAAACAGCCATCTTA-3' \\
\hline mouse Cathepsin K & 5'-CGAAAAGAGCCTAGCGAACA-3' 5'-TGGGTAGCAGCAGAAACTTG-3' \\
\hline mouse Cathepsin L & 5'-CAAATAAGAATAAATATTGGCTTGTCA-3' 5' -TGTAGCCTTCCATACCCCATT-3' \\
\hline mouse CTGF & 5'-TGACCCCTGCGACCCACA-3' 5'-TACACCGACCCACCGAAGACACAG-3' \\
\hline mouse $\beta$-MHC & 5'-ATGTGCCGGACCTTGGAA-3' 5'-CCTCGGGTTAGCTGAGAGATCA-3' \\
\hline mouse $\alpha$-SMA & 5'-TCCAGCCATCTTTCATTGGGA-3' $5^{\prime}$-CCCCTGACAGGACGTTGTTA-3' \\
\hline mouse COL1 $\alpha 1$ & 5'-GAGCGGAGAGTACTGGATCG-3' 5'-GTTCGGGCTGATGTACCAGT-3' \\
\hline mouse GAPDH & 5'-ACCCAGAAGACTGTGGATGG-3' 5'-CACATTGGGGGTAGGAACAC-3' \\
\hline
\end{tabular}

Table 2. Primers for PCR.

despite elevating blood pressure similarly to Ang II infusion. This suggested that the effects of Ang II on triggering TAD onset were not caused by a blood pressure change alone ${ }^{8}$. To further support this, we found that $87 \%$ mice treated only with BAPN developed thoracic aortic dissection and $37 \%$ had spontaneous rupture without elevation of systolic blood pressure. As expected, thoracic aortic dissection and rupture were observed in $100 \%$ of mice receiving both BAPN and Ang II on both genetic backgrounds, supporting a triggering effect of Ang II after aortic structural disruption by BAPN. Loss of elasticity resulted in decreased diastolic blood pressure ${ }^{16}$, explaining our observation of reduced diastolic blood pressure in mice of both genetic backgrounds given BAPN alone, as compared with controls. Interestingly, spontaneous rupture occurred with mice given the lower doses, but not the higher doses, of BAPN. These mice also showed limited weight increases over time, suggesting that a sufficient hemodynamic load was required for the progression of TAD. Further investigation will be needed to understand the mechanisms involved in this mouse model for BAPN-induced TAD.

\section{Methods}

Patient specimens and ethics statement. Dissection samples were collected from TAD patients who had undergone repair surgery in Beijing Anzhen Hospital. None of the patients had a known genetic syndrome related to aortic diseases, such as Marfan's, Turner's, Loeys-Dietz or Ehlers-Danlos syndromes. Control aortas were trimmings that had been discarded during heart transplantation surgeries. Informed consent was obtained for use of these specimens. Use of human tissue was approved by the Medical Ethical Committee of Capital Medical University, Beijing, and complied with the principles outlined in the Declaration of Helsinki ${ }^{4}$.

Animal model and ethics statement. Wildtype C57BL/6 and FVB mice were obtained from HFK Bioscience Company (Beijing, China). All studies were reviewed and approved by The Institutional Animal Care and Use Committee of Capital Medical University, Beijing, China. The investigation conformed to the Guide for the Care and Use of Laboratory Animals, published by the US National Institutes of Health (NIH). Three-week-old male mice were fed a normal diet and administered freshly prepared BAPN (Sigma-Aldrich, St. Louis, MO, USA) solution dissolved in the drinking water $(1 \mathrm{~g} / \mathrm{kg} / \mathrm{d})$ for $4 \mathrm{wk}$, as described previously ${ }^{8}$. Blood pressure was measured before and after BAPN administration for $4 \mathrm{wk}$, using the tail-cuff method. Interventions lasted $4 \mathrm{wk}$ and body weights were measured weekly. As previously reported, at $7 \mathrm{wk}$ old, osmotic mini pumps (Alzet, Cupertino, CA, USA) administering $1 \mu \mathrm{g} / \mathrm{kg}$ per min Ang II (Sigma-Aldrich, St. Louis, MO, USA) were implanted subcutaneously and mice were euthanized $24 \mathrm{~h}$ after implantation ${ }^{17}$. All mice died before expected end time of the experiment were autopsied immediately, and Blood clots were found in the thoracic cavities of these mice. Mice surviving at the end of the experiment were sacrificed by an overdose of sodium pentobarbital and their blood and tissue samples were collected for further analyses.

Histopathological analysis. Complete gross and histopathological evaluations were performed with samples from control and BAPN-treated mice. After euthanasia, normal and dissected aortas were harvested from the ascending aorta to the iliac artery and were fixed in $10 \%$ buffered formalin, as were human tissues. Fixed, paraffin-embedded tissues were cut at $5 \mu \mathrm{m}$ thickness, stained with haematoxylin and eosin following standard procedures and examined under light microscopy, as previously described ${ }^{4}$.

Elastin staining. Elastin in the normal and dissected aortas was stained with the Gomrori's aldehyde-fuchsin staining method, using an elastic fibre staining kit (Maixin Bio, Fuzhou, China) as previously described ${ }^{4}$. Briefly, after deparaffinization and rehydration, sections were incubated for $5 \mathrm{~min}$ in Lugol's iodine solution, washed with PBS and incubated with sodium thiosulfate for $5 \mathrm{~min}$. After washing with PBS and $70 \%$ ethanol, sections were incubated with aldehyde-fuchsin for $10 \mathrm{~min}$ and acid Orange $\mathrm{G}$ for $\mathrm{s}$.

Plasma lipid measurements. Plasma levels of triglyceride (TG) and cholesterol (CHO) were measured by COD-PAP and GPO-PAP methods, respectively, with automated clinical chemistry analyser kits (Biosino Biotech, Beijing, China). 
Quantitative real-time polymerase chain reaction (RT-PCR). Total RNA of aortic media without thrombi was isolated by the acid-phenol extraction method in the presence of chaotropic salts (Trizol, Thermo Fisher, Carlsbad, CA, USA) and subsequent isopropanol-ethanol precipitation, as described previously ${ }^{18}$. RNA $(2 \mu \mathrm{g})$ was reverse-transcribed using the GoScript ${ }^{\mathrm{TM}}$ Reverse Transcription System (Thermo Fisher, Carlsbad, CA, USA), according to the manufacturer's instructions. For real-time quantitative PCR, the iQ5 system (Bio-Rad, Hercules, CA, USA) with SYBR Green I (Takara, Shiga, Japan) was used. Amplification was performed at $95^{\circ} \mathrm{C}$ for $5 \mathrm{~min}, 95^{\circ} \mathrm{C}$ for $45 \mathrm{~s}$ and $60^{\circ} \mathrm{C}$ for $1 \mathrm{~min}$, for $45 \mathrm{cycles}$. The housekeeping gene glyceraldehyde 3-phosphate dehydrogenase (GAPDH) was used as a control. The primers used are listed in Table 2.

Statistical analysis. In all experiments, data were collected form more than 6 mice per group to calculate means \pm standard deviation (SD). Student's t-test was used for statistical analysis and $P<0.05$ was considered statistically significant.

\section{References}

1. Wu, D., Shen, Y. H., Russell, L., Coselli, J. S. \& LeMaire, S. A. Molecular mechanisms of thoracic aortic dissection. The Journal of surgical research 184, 907-924 (2013).

2. Anzai, A. et al. Adventitial CXCL1/G-CSF expression in response to acute aortic dissection triggers local neutrophil recruitment and activation leading to aortic rupture. Circulation research 116, 612-623 (2015).

3. Nienaber, C. A. \& Clough, R. E. Management of acute aortic dissection. The Lancet 385, 800-811 (2015).

4. Jia, L. X. et al. Mechanical stretch-induced endoplasmic reticulum stress, apoptosis and inflammation contribute to thoracic aortic aneurysm and dissection. The Journal of pathology 236, 373-383 (2015).

5. Wang, S. et al. Activation of AMP-activated protein kinase alpha2 by nicotine instigates formation of abdominal aortic aneurysms in mice in vivo. Nature medicine 18, 902-910 (2012).

6. Pagano, M. B. et al. Complement-dependent neutrophil recruitment is critical for the development of elastase-induced abdominal aortic aneurysm. Circulation 119, 1805-1813 (2009).

7. Gopal, K., Kumar, K., Nandini, R., Jahan, P. \& Kumar, M. J. High fat diet containing cholesterol induce aortic aneurysm through recruitment and proliferation of circulating agranulocytes in apoE knock out mice model. Journal of thrombosis and thrombolysis $\mathbf{3 0}$, 154-163 (2010).

8. Kurihara, T. et al. Neutrophil-derived matrix metalloproteinase 9 triggers acute aortic dissection. Circulation 126, 3070-3080 (2012).

9. Maki, J. M. Inactivation of the Lysyl Oxidase Gene Lox Leads to Aortic Aneurysms, Cardiovascular Dysfunction, and Perinatal Death in Mice. Circulation 106, 2503-2509 (2002).

10. Ebony Washington Remus et al. The role of lysyl oxidase family members in the stabilization of abdominal aortic aneurysms. Am J Physiol Heart Circ Physiol 303, H1067-1075 (2012).

11. Ghosh, A., Pechota, A., Coleman, D., Upchurch, G. R. Jr. \& Eliason, J. L. Cigarette smoke-induced MMP2 and MMP9 secretion from aortic vascular smooth cells is mediated via the Jak/Stat pathway. Human pathology 46, 284-294 (2015).

12. Zhang, X. et al. Matrix metalloproteinase levels in chronic thoracic aortic dissection. The Journal of surgical research 189, 348-358 (2014).

13. Lv, B. J., Lindholt, J. S., Wang, J., Cheng, X. \& Shi, G. P. Plasma levels of cathepsins L, K, and V and risks of abdominal aortic aneurysms: a randomized population-based study. Atherosclerosis 230, 100-105 (2013).

14. Elena, M. Gallo et al. Angiotensin II-dependent TGF- $\beta$ signaling contributes to Loeys-Dietz syndrome vascular pathogenesis. J Clin Invest 124, 448-460 (2014).

15. Goldfinger, J. Z. et al. Thoracic aortic aneurysm and dissection. Journal of the American College of Cardiology 64, 1725-1739 (2014).

16. El-Hamamsy, I. \& Yacoub, M. H. Cellular and molecular mechanisms of thoracic aortic aneurysms. Nature Reviews Cardiology 6, 771-786 (2009)

17. Shimizu-Hirota, R. et al. Extracellular matrix glycoprotein biglycan enhances vascular smooth muscle cell proliferation and migration. Circulation research 94, 1067-1074 (2004).

18. Fattori, R. et al. Interdisciplinary expert consensus document on management of type B aortic dissection. Journal of the American College of Cardiology 61, 1661-1678 (2013).

\section{Acknowledgements}

This work was supported by grants from the Chinese Ministry of Science and Technology (grant number 2012CB945104), National Natural Science Foundation of China (grant numbers 81300120, 81230006), Beijing Nova Program (grant number Z151100000315067), Beijing Natural Science Foundation (grant number 7142030,7162030) and Beijing Collaborative Innovative Research Center for Cardiovascular Diseases (PXM2014_014226_000002). We would like to thank Wenjian Jiang and Hongjia Zhang for preparing the human samples and Fujian Wu and Hongxia Li for their generous support and encouragement during this study.

\section{Author Contributions}

W.R., Y.L. and J.D. designed the study; W.R., Y.L. and X.W. performed the experiments; W.R., Y.L., X.W., C.P. and L.J. performed data analysis; W.R., Y.L., F.L. and J.D. wrote the manuscript; and J.D. approved the final manuscript.

\section{Additional Information}

Competing financial interests: The authors declare no competing financial interests.

How to cite this article: Ren, W. et al. $\beta$-Aminopropionitrile monofumarate induces thoracic aortic dissection in C57BL/6 mice. Sci. Rep. 6, 28149; doi: 10.1038/srep28149 (2016).

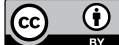

This work is licensed under a Creative Commons Attribution 4.0 International License. The images or other third party material in this article are included in the article's Creative Commons license, unless indicated otherwise in the credit line; if the material is not included under the Creative Commons license, users will need to obtain permission from the license holder to reproduce the material. To view a copy of this license, visit http://creativecommons.org/licenses/by/4.0/ 